\title{
DIAGNÓSTICO DEL SISTEMA DE APROVECHAMIENTO DEL AGUA LLUVIA EN EL CONSEJO COMUNITARIO DE LA COMUNIDAD NEGRA DE LOS LAGOS, BUENAVENTURA
}

\author{
Nixon Arboleda Montaño
}

Recibido el 10 de marzo de 2015, aprobado el 7 de julio de 2015 y actualizado el 12 de mayo de 2016

\section{DOI: 10.17151/luaz.2016.43.3}

\section{RESUMEN}

Este trabajo presenta los resultados de la investigación realizada en el Consejo Comunitario de la Comunidad Negra Los Lagos (CCCN Los Lagos); la cual consistió en diagnosticar las condiciones de aprovechamiento del agua lluvia, por lo que se pudo determinar el potencial de aprovechamiento del agua lluvia en la comunidad, los tipos de techos que utilizan las viviendas, el sistema de recolección y conducción del agua lluvia, los tipos de recipientes para realizar el almacenamiento del agua lluvia. Para la identificación de los aspectos anteriores, se realizaron visitas al lugar, así como también la realización de entrevistas semiestructuradas y encuestas (anexo 1).

Luego de conocer el sistema de abastecimiento del agua lluvia, se realizó análisis fisicoquímicos y microbiológicos al agua lluvia recolectada, permitiendo conocer con ello el cumplimiento o no de la normativa ambiental; además, se aplicaron análisis estadísticos para determinar correlaciones y varianzas en los resultados.

Palabras clave: almacenamiento de agua, captación de agua lluvia, calidad del agua.

\section{DIAGNOSTIC SYSTEM RAIN WATER HARVESTING IN THE BLACK COMMUNITY COUNCIL COMMUNITY OF LAGOS, BUENAVENTURA}

\section{ABSTRACT \\ This paper presents the results of research conducted at the Community Council of the black community of Los Lagos, which was to diagnose the conditions of use of}


rain water, so they could determine the potential use of rain water in the community, the types of roofs used housing, the system of collection and conveyance of rain water, types of containers for storage of rain water; for the identification of the above aspects, site visits were conducted, as well as semistructured interviews and surveys. After meeting the supply system of rain water, chemical and microbiological to rain water collected analyzes were performed, thus allowing to know or not compliance with environmental regulations; Additional statistical analyzes were applied to determine correlations and variances in the results.

Keywords: Water storage, rainwater harvesting, water quality.

\section{INTRODUCCIÓN}

Actualmente alrededor de 1.100 millones de personas no tiene acceso al agua tratada y más de 2.600 millones de personas carecen de servicios de saneamiento básico. La insuficiencia de agua afecta a cerca de 2.000 millones de personas en el mundo (Guhl, 2007). Si no se toman medidas para revertir la tendencia, una de cada tres personas estará viviendo en un país con escasez de agua potable. Del mismo modo, si bien el problema afecta a todos los países, los más perjudicados son los de las economías más pobres, donde nace el 95\% de las 80 millones de personas que cada año incrementan la población del planeta (Duran, Op Cit).

Se prevé que en los próximos veinte años el promedio mundial de abastecimiento de agua por habitante disminuirá en un tercio a causa del crecimiento de la población, de la contaminación y del cambio climático. A mediados de este siglo habrá 2000 millones de personas en 48 países que sufrirán de escasez de agua; el peor de los escenarios muestra a 7000 millones de personas en 60 países con ese problema (Duran, Op Cit).

Por su parte en Colombia, de acuerdo con el DANE (2013), el abastecimiento de agua presentó una cobertura de acueducto total (rural y urbano), en el año 2012 del $75,15 \%$. En el caso del sector urbano para este mismo año tuvo una cobertura del $97 \%$ y el rural $53,3 \%$. Lo anterior representa buenos niveles de cobertura de acueducto en las viviendas urbanas; no obstante, solo alrededor de la mitad de las viviendas del sector rural de Colombia presentan cobertura de acueducto, 
evidenciando una alta disparidad en la cobertura de agua potable entre las cabeceras y las zonas rurales.

La región pacífica se caracteriza por presentar bajos niveles de cobertura de agua potable en sus áreas rurales y urbanas, incluso muy por debajo del promedio nacional, ya que de acuerdo con el Departamento Nacional de Planeación (2007) tan sólo alcanza una cobertura general del $42,8 \%$, siendo posible encontrar que en muchos municipios no existe corregimiento o vereda alguna con un sistema de acueducto, o a la sumo se encuentran en muy baja cantidad, alcanzando porcentajes muy por debajo de los promedios nacionales. Esta situación conduce a que las personas presenten inadecuadas condiciones de vida, sobre todo en el campo de la salud, pues la falta de saneamiento en el agua es escasa, lo que da paso al desarrollo de virus y bacterias incrementando la morbilidad y mortalidad en la población.

Ante esta situación de las zonas rurales de la región pacífica, el agua lluvia ha sido para muchas de las comunidades que allí habitan la principal fuente de abastecimiento de agua, dado los altos índices de precipitación, convirtiéndola en la región más lluviosa de Colombia y una de las más lluviosas en el mundoii.

De esta forma, las aguas lluvias representan un recurso importante para las comunidades de la zona rural en la región pacífica de Colombia, recurso del cual pueden disponer sin la necesidad de recorrer grandes distancias, merced a los sistemas tradicionales de abastecimiento que actualmente se tienen (Centro Internacional de Demostración y Capacitación en Aprovechamiento del Agua de Lluvia, 2007).

\section{METOdOLOGÍA}

\section{1. Área de estudio}

El Consejo Comunitario de la Comunidad Negra de Los Lagos es una comunidad rural con un territorio relativamente plano, que tiene una figura de administración colectiva, en la cual todos son dueños del territorio salvo el espacio que cada familia históricamente ha trabajado ${ }^{i i i}$. A este lugar, se puede acceder por vía marítima o terrestre y está localizado en la zona continental del distrito de 
Buenaventura, cuenca baja del rio Dagua, sobre la Vía Simón Bolívar hacia al aeropuerto, a la altura del kilómetro quince.

Esta comunidad carece de muchos servicios públicos, tal es el caso del acueducto, alcantarillado, gas natural, telefonía fija y aseo; contando solamente con energía eléctrica.

En la actualidad, el Consejo Comunitario de la Comunidad Negra Los Lagos cuenta con 68 familias que se representan en más de 412 habitantes, los cuales están distribuidos de la sigue forma:

Tabla 1. Distribución de la población en el Consejo Comunitario de la Comunidad Negra Los Lagos

\begin{tabular}{|c|c|c|c|}
\hline Rangos & Hombre & Mujeres & Total \\
\hline Rango $0-7$ años & 41 & 63 & 104 \\
\hline Rango $8-17$ años & 86 & 27 & 113 \\
\hline 18 años en adelante & 102 & 93 & 195 \\
\hline Total & & 412 \\
\hline
\end{tabular}

Fuente: propia con base en Acta de registro No 48 del 8 de noviembre de 2010 de la Alcaldia Distrital de Buenaventura

El tipo de poblamiento es permanente y se tienen fincas o espacios productivos de pan coger en las partes traseras de las viviendas.

\subsection{Método de estudio}

Estudio exploratorio y descriptivo, ya que en Buenaventura y la zona del pacifico no se han realizado estudios de esta naturaleza. (Hurtado, 2000; 2002).

\subsection{Tipo de estudio}

El estudio se enmarcó de tipo cuantitativo y cualitativo.

\subsection{Materiales y métodos}

\subsubsection{Recorridos de campo, encuestas y entrevistas}

Con la finalidad de conocer algunas características esenciales de las viviendas de la zona de estudio, su composición, formas de captación y usos del agua lluvia; se 
realizaron recorridos de campo y aplicaron encuestas a los jefes de hogar y representantes o líderes de la comunidad.

Con esta finalidad, se realizó un censo total a las 68 viviendas que conforman la zona de estudio, aplicando la encuesta (anexo 1) a los jefes de hogar.

\subsubsection{Análisis de la calidad del agua lluvia captada}

Para el análisis de la calidad del agua lluvia captada, en total se evaluaron 3 meses, comprendidos desde julio hasta septiembre del año 2013.

La metodología para el análisis de la calidad de las aguas lluvias en el CCCN Los Lagos se presenta en la figura a continuación.

Figura 1. Metodologia para el análisis de la calidad de las aguas lluvias en el CCCN Los Lagos

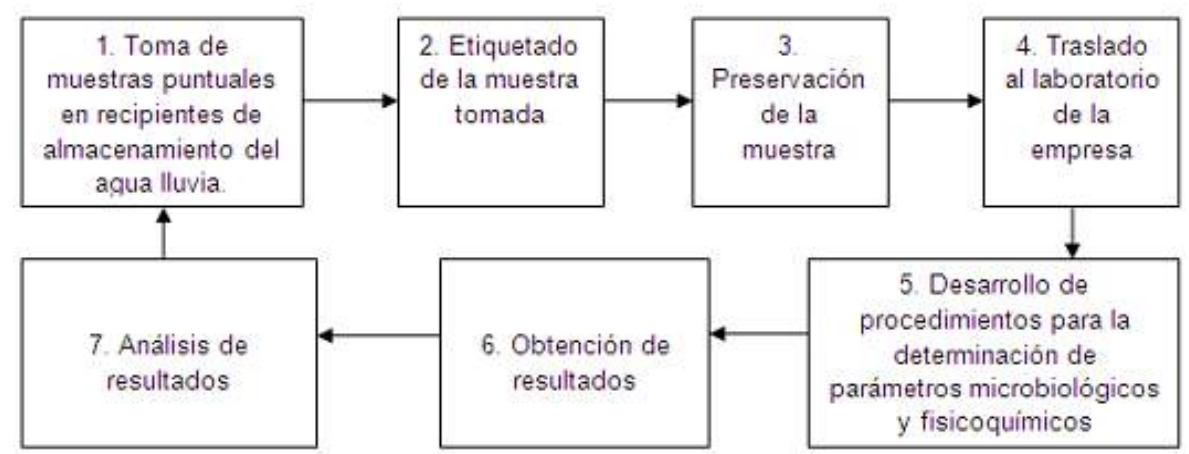

Fuente: propia con base en Organización Panamericana de la Salud, 2010.

En el caso del análisis microbiológico se evaluaron los parámetros establecidos en la tabla 2, de acuerdo como se presenta a continuación:

Tabla 2. Parámetros a evaluar en el análisis microbiológico de la calidad del agua lluvia.

\begin{tabular}{|l|c|c|c|}
\hline \multicolumn{4}{|c|}{ ANALISIS MICROBIOLOGICO } \\
\hline PARAMETROS & EXPRESADO COMO & METODO UTILIZADO & $\begin{array}{c}\text { VALOR } \\
\text { OBTENIDO }\end{array}$ \\
\hline Coliformes totales & 0 microorganismos/100 & $\begin{array}{c}\text { Filtración por membrana } \\
\text { con chromocult }\end{array}$ & \\
\hline Eschericha coli & 0 microorganismos/100 & $\begin{array}{c}\text { Filtración por membrana } \\
\text { con chromocult }\end{array}$ & \\
\hline
\end{tabular}

Fuente: propia basado en el decreto 1575 de 2007 y resolución 2115 de 2007 
Para la toma de muestras, se procedió de la siguiente manera:

El área de estudio cuenta con 68 viviendas, dicha área se dividió estratégicamente en 5 zonas: esquinas y centro. En cada zona se seleccionaron aleatoriamente 2 viviendas; 1 con techo de zinc y 1 con techo de asbesto, preferiblemente aquellas que presentaban mayores niveles de captación de aguas lluvias. En total se seleccionaron 10 viviendas.

De esta forma, para el análisis microbiológico, en cada una de las viviendas seleccionadas, mensualmente se tomó una muestra por un término de tiempo de tres meses, por lo que en total se tomaron 30 muestras, ya que cada mes se realizó el mismo ejercicio para las 10 viviendas seleccionadas. La toma de muestra consistió en la recolección de 0,2L de agua de los recipientes de almacenamiento del agua lluvia haciendo uso de un frasco de vidrio transparente, guantes y tapabocas (anexo 2), seguidamente se llevaron al laboratorio de la empresa Hidropacífico E.S.P S.A $A^{\text {iv }}$, en la cual se realizaron los respectivos procedimientos con base en los métodos normalizados para el análisis de aguas potables y residuales (APHA WWA, 1992 citado por Severiche; Castillo \& Acevedo, 2013; Organización Mundial de la Salud, 2006).

Los resultados se promediaron, obteniéndose un registro por mes para cada uno de los parámetros evaluados.

Por su parte, en el caso del análisis físico-químico se evaluaron los parámetros establecidos en la tabla 3, de acuerdo como se presenta a continuación: 
Tabla 3. Parámetros a evaluar en el Análisis físico-químico de la calidad del agua lluvia.

\begin{tabular}{|c|c|c|c|c|c|}
\hline \multicolumn{6}{|c|}{ ANALISIS FISICO - QUIMICO } \\
\hline \multicolumn{2}{|c|}{ PARAMMTROS MEDIDOS } & EXPRESADO & METODO & OBTENIDO & Cumple \\
\hline $\begin{array}{l}\text { Sustancias } \\
\text { flotantes }\end{array}$ & $\begin{array}{l}\text { Unidad } \\
\text { medida }\end{array}$ & Ausencia & $\begin{array}{c}\text { Organoleptico } \\
\text { (visual) }\end{array}$ & & \\
\hline Olor & & Aceptable & $\begin{array}{c}\text { Organoleptico } \\
\text { (olfato) }\end{array}$ & & \\
\hline Sabor & & Aceptable & $\begin{array}{c}\text { Organoleptico } \\
\text { (gusto) }\end{array}$ & & \\
\hline Turbiedad & U.N.T. & $\bar{z}=2.0 \mathrm{U} . \mathrm{N} . \mathrm{T}$ & $\begin{array}{c}\text { Turbidimetro } \\
\text { (marca HACH } \\
\text { Modelo 2100P) }\end{array}$ & & \\
\hline Color & PLCO & $\bar{z}=150 \mathrm{PLCO}$ & $\begin{array}{c}\text { Espectrofotometro } \\
\text { (marca HACH } \\
\text { ModeloDR 2800) }\end{array}$ & & \\
\hline $\mathrm{pH}$ & UNpH & $\bar{z}=6.5 \mathrm{z}=9.0 \mathrm{UN}$ & ModelPHC10101 & & \\
\hline Conductividad & $\mathrm{ms} / \mathrm{cm}$ & $\bar{z}=1500 \mathrm{~ms} / \mathrm{cm}$ & $\begin{array}{l}\text { Conductimetria } \\
\text { (electrodo) }\end{array}$ & & \\
\hline Acidez & $\begin{array}{c}\mathrm{mg} / \mathrm{LCa} \\
\mathrm{Co} 3\end{array}$ & $\bar{z}=50 \mathrm{mg} / \mathrm{L}$ & $\begin{array}{c}\text { Titulacion } \\
\text { potenciométrica }\end{array}$ & & \\
\hline Dureza total & $\begin{array}{c}\mathrm{mg} / \mathrm{LCa} \\
\mathrm{Co} 3\end{array}$ & $\bar{z}=300 \mathrm{mg} / \mathrm{L}$ & $\begin{array}{c}\text { Titulacion con } \\
\text { EDTA }\end{array}$ & & \\
\hline Sulfatos & $\mathrm{mg} / \mathrm{LSO} 4$ & $\bar{z}=250 \mathrm{mg} / \mathrm{L}$ & $\begin{array}{l}\text { Espectrofotometro } \\
\text { (marca HACH } \\
\text { Modelo DR 2800 }\end{array}$ & & \\
\hline Cloruros & $\mathrm{mg} / \mathrm{LCl}$ & $\bar{z}=250 \mathrm{mg} / \mathrm{L}$ & $\begin{array}{l}\text { Titulometrico con } \\
\text { nitrato mercúrico }\end{array}$ & & \\
\hline Nitritos & $\begin{array}{c}\mathrm{mg} / \mathrm{L} \\
\mathrm{NHNO} 2\end{array}$ & $\bar{z}=0.1 \mathrm{mg} / \mathrm{L}$ & $\begin{array}{l}\text { Espectrofotometro } \\
\text { (marca HACH } \\
\text { Modelo DR 2800 }\end{array}$ & & \\
\hline Nitratos & $\begin{array}{l}\mathrm{mg} / \mathrm{L} \\
\mathrm{NHNO} 3\end{array}$ & $\bar{z}=10 \mathrm{mg} / \mathrm{L}$ & $\begin{array}{l}\text { Espectrofotomedro } \\
\text { (marca HACH } \\
\text { Modelo DR 2800 }\end{array}$ & & \\
\hline Hierro total & $\mathrm{mg} / \mathrm{LF} 63+$ & $\bar{z}=0.3 \mathrm{mg} / \mathrm{L}$ & $\begin{array}{l}\text { Espectrofotometo } \\
\text { (marca HACH } \\
\text { Modelo DR } 2800\end{array}$ & & \\
\hline $\begin{array}{l}\text { Aluminio } \\
\text { residual }\end{array}$ & $\mathrm{mg} / \mathrm{LA}$ & $\bar{z}=0.2 \mathrm{mg} / \mathrm{L}$ & $\begin{array}{l}\text { Espectrofotometro } \\
\text { (marca HACH } \\
\text { Modelo DR } 2800\end{array}$ & & \\
\hline $\begin{array}{c}\text { Cloro residual } \\
\text { libre }\end{array}$ & $\mathrm{mg} / \mathrm{LC} 2$ & $\bar{z}=0.3 \bar{z}=2.0$ & $\begin{array}{l}\text { Thedidor de cloro } \\
\text { HACH POCKET II }\end{array}$ & & \\
\hline
\end{tabular}

Para el análisis físico-químico, al igual que en el análisis microbiológico en cada una de las viviendas seleccionadas, mensualmente se tomó una muestra por un término de tiempo de tres meses. La toma de muestra consistió en la recolección de $1 \mathrm{~L}$ de agua de los recipientes de almacenamiento del agua lluvia haciendo uso de un envase plástico, guantes y tapabocas (anexo 2), seguidamente se llevaron al laboratorio de la empresa Hidropacífico E.S.P S.A, en la cual se realizaron los respectivos procedimientos con base en los métodos normalizados para el análisis de aguas potables y residuales (APHA WWA, 1992 citado por Severiche; Castillo \& Acevedo, 2013).

Los resultados se promediaron, obteniéndose un registro por mes para cada uno de los parámetros evaluados. 


\section{RESULTADOS}

\subsection{Sistema de aprovechamiento del agua lluvia}

El sistema de aprovechamiento del agua lluvia presenta el potencial de aprovechamiento del agua lluvia, propiedad de las viviendas y promedio de personas que la habitan, captación del agua lluvia, recolección y conducción del agua lluvia, y almacenamiento del agua lluvia.

\subsubsection{Potencial de aprovechamiento del agua lluvia}

En la tabla a continuación se presentan los resultados de precipitación ${ }^{\vee}$ en el CCCN Los Lagos.

Tabla 4. Promedio mensual de precipitaciones en el CCCN Los Lagos

\begin{tabular}{|c|c|c|c|}
\hline Mes & Precipitacion (mm) & Mes & Precipitacion \\
\hline Enero & 253,6 & Julio & 545,3 \\
\hline Febrero & 302,9 & Agosto & 768,0 \\
\hline Marzo & 401,6 & Septiembre & 829,2 \\
\hline Abril & 395,8 & Octubre & 960,1 \\
\hline Mayo & 647,0 & Noviembre & 765,1 \\
\hline Junio & 589,4 & Diciembre & 815,0 \\
\hline Fuente: propia con base en datos de precipitación de estación meteorológica del IDEAM, 2014
\end{tabular}

De acuerdo con la tabla anterior, se puede determinar que en el CCCN Los Lagos se cuenta con un alto potencial de aprovechamiento de agua lluvia; sin embargo, en las viviendas se cuenta con poca capacidad para almacenar este recurso, convirtiéndose en la causa de desabastecimiento de agua en la mayoría de los hogares de esta comunidad cuando cesa de llover más de una semana.

\subsubsection{Propiedad de las viviendas y promedio de personas que la habitan}

Respecto a la propiedad de las viviendas, es relevante destacar que el $100 \%$ de las viviendas son propias de las familias que las habitan; sin embargo, el rango de personas que en promedio habitan una vivienda es significativo, tal como se indica en la siguiente figura. 
Figura 2. Rango de personas que habitan las viviendas

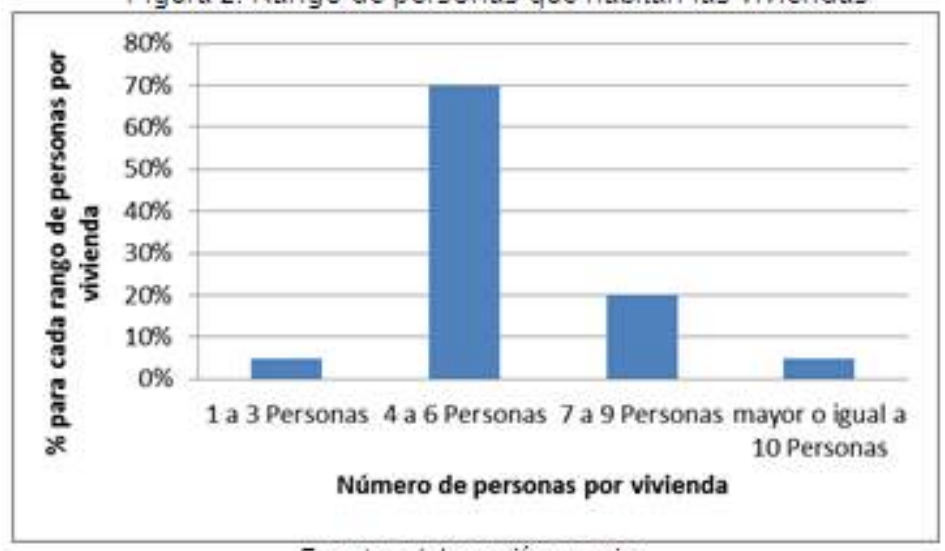

Fuente: elaboración propia

El $70 \%$ de las viviendas están habitadas entre 4 y 6 personas; por su parte, el $20 \%$ de los hogares están habitados por un número que oscila entre 7 y 9 personas; los casos de menor porcentaje de ocupación se presentan en los rangos entre 1 y 3 personas por vivienda y el mayor o igual a 10 personas por vivienda, representando cada uno de ellos tan sólo un 5\%. Lo anterior indica que en el Consejo Comunitario se está presentando un alto índice de ocupación en las viviendas, lo que es normal en las zonas rurales del Distrito de Buenaventura; sin embargo, ello repercute significativamente en el aprovechamiento del recurso hídrico dado que a mayor número de personas se requiere mayor volumen de agua para satisfacer las necesidades domésticas.

Para satisfacer sus necesidades de agua para uso doméstico u otros usos, esta comunidad no cuenta con sistema de acueducto, siendo el agua lluvia su principal fuente de abastecimiento, encontrándose en un segundo renglón el agua superficial representada en los ríos y las quebradas que irrigan el lugar; respecto al agua subterránea no existe ningún tipo de aprovechamiento puesto que en el lugar no se ha identificado alguna fuente de este tipo.

Dado que el agua lluvia es la principal fuente abastecedora, en todas las viviendas se realiza aprovechamiento o cosecha de estas; destacando como elementos comunes del sistema de captación los siguientes: área captación; recolección y conducción y almacenamiento. 


\subsubsection{Captación del agua lluvia}

El área de captación está conformada básicamente por el techo de las viviendas, el cual no se compone del mismo material, tal como se expresa en la figura 3.

\section{Figura 3. Materiales que se utilizan en los techos de las viviendas}

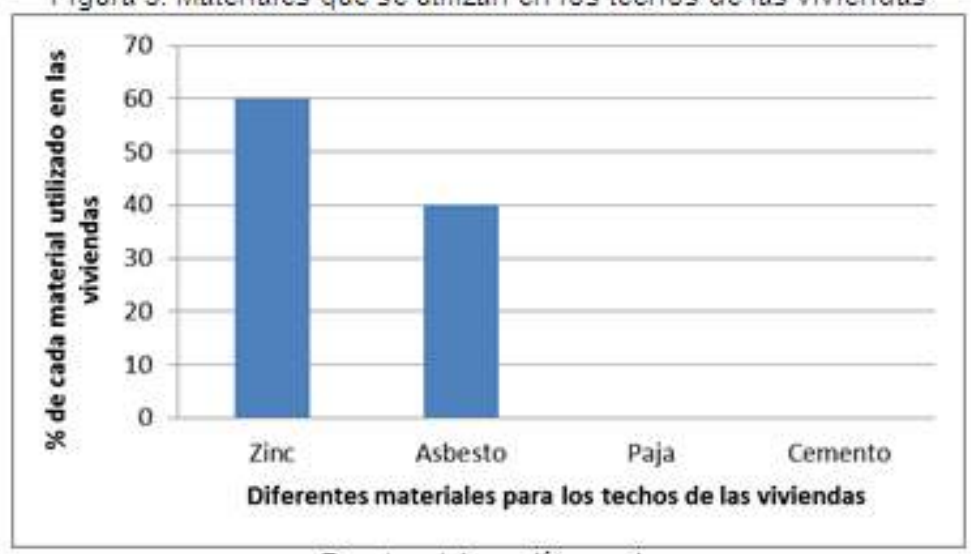

Fuente: elaboración propia.

De acuerdo con los resultados obtenidos, en el Consejo Comunitario se utilizan dos tipos de materiales para los techos de las viviendas, siendo el zinc el que más se utiliza con un $60 \%$ seguido del asbesto con un $40 \%$. De otra parte, no se presenta el uso de ningún otro material en la zona.

En figuras 4 y 5 se observan los materiales utilizados para los techos de las viviendas.

Figura 4. Techo en zinc utilizado en las viviendas

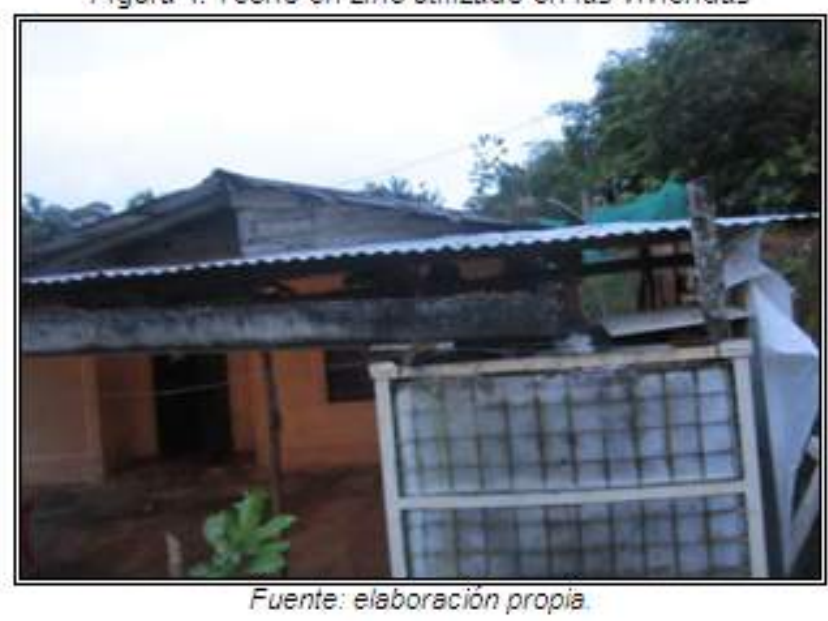


En términos generales los techos de zinc presentan un buen estado, sin presencia de material vegetal, ni otros elementos permanentes u ocasionales sobre el mismo, indicando que es un material muy apto para ser utilizado en el lugar.

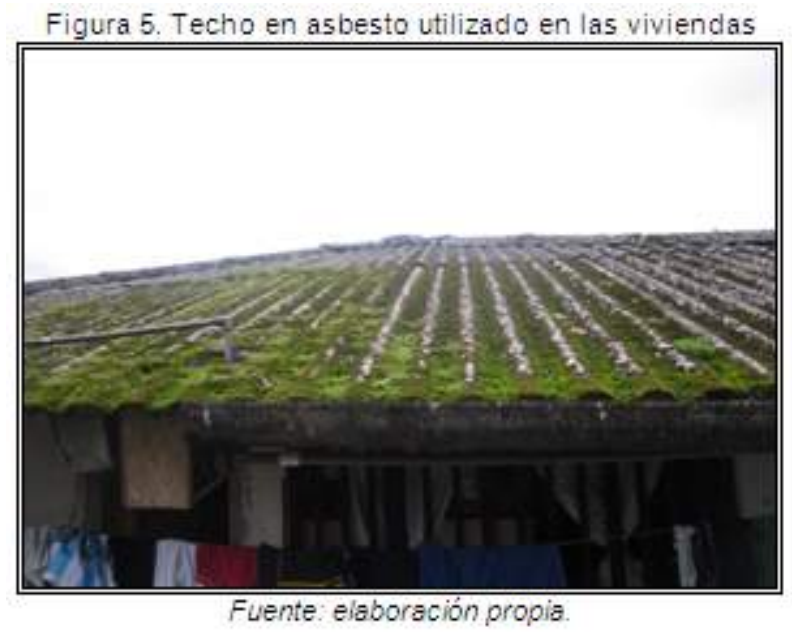

Es evidente que los techos de asbesto no presentan un buen estado, dado que se observa sobre sí mismo mucha presencia de material permanente como musgos, y otros elementos como restos de hojas, madera y demás, situación que implica un gran esfuerzo para limpiar este techo con la dificultad de que luego se volvería a ensuciar.

\subsubsection{Recolección y conducción del agua lluvia}

En lo que respecta a la recolección y conducción del agua lluvia, se reportaron los mismos materiales utilizados en los techos (zinc y asbesto) en la misma proporción de utilización. Las figuras 35 y 36 presentan estos materiales. 


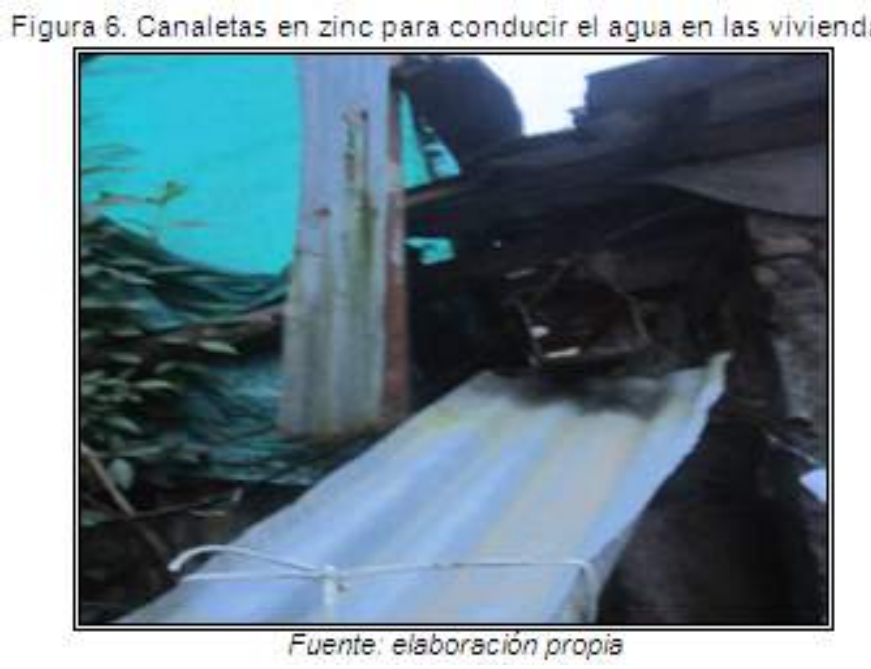

Las canaletas en zinc presentan un buen estado de limpieza, sin presencia de material vegetal, ni otros elementos permanentes $u$ ocasionales sobre el mismo.

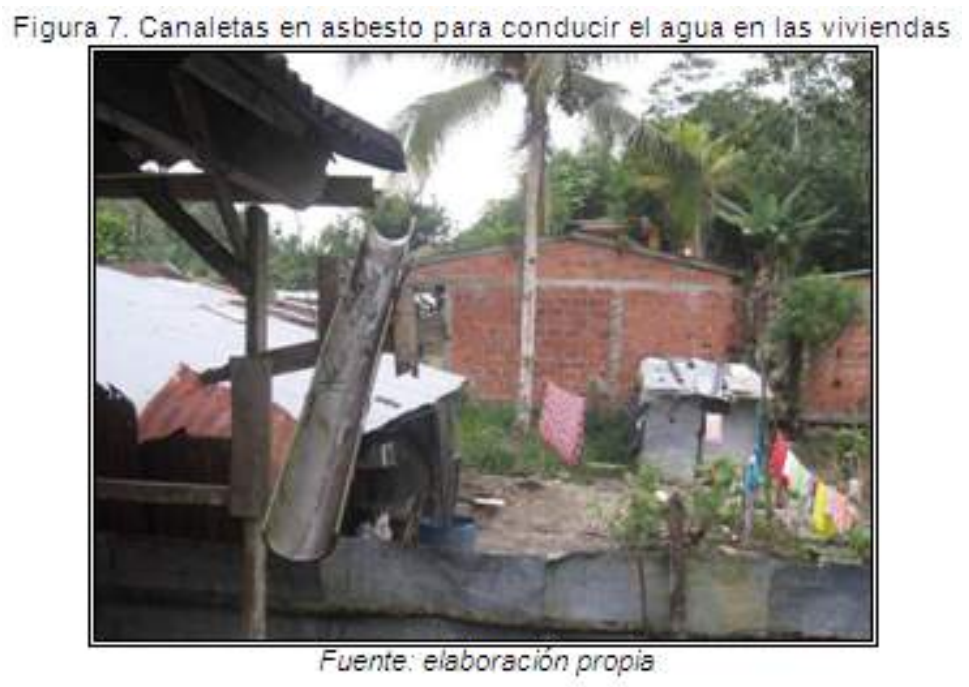

Las canaletas en asbesto a diferencia de las canaletas de zinc no presentan un buen estado de limpieza, puesto que se observa en ellas presencia de mugre y lama.

\subsubsection{Almacenamiento del agua lluvia}


Por su parte, el almacenamiento del agua lluvia se lleva a cabo en recipientes variados, tales como baldes de pequeña capacidad, galonetas, tanques de 500 y 1000 litros hasta pilas, tal como se muestra en las fotos a continuación.

Figura 8. Diferentes recipientes plásticos para el almacenamiento del agua lluvia.

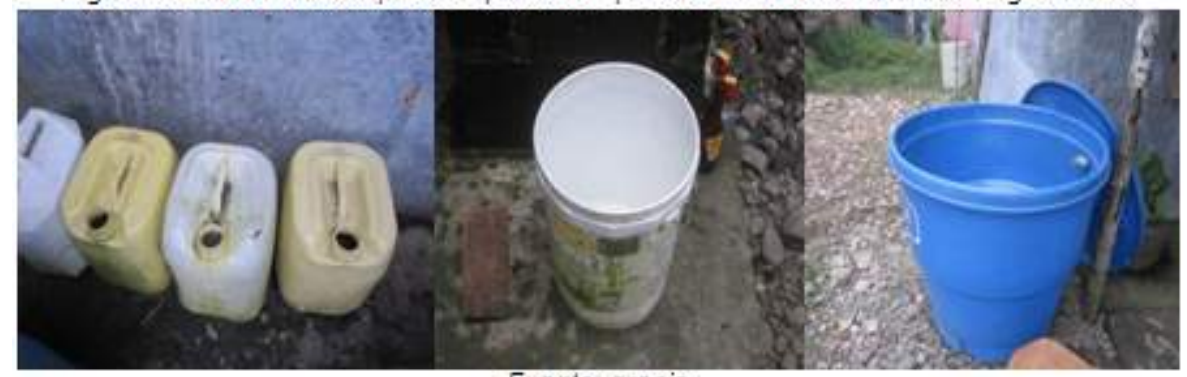

Fuente propia.

La Figura 8 presenta diferentes envases plásticos utilizados para almacenar agua en las diferentes viviendas del Consejo Comunitario.

Figura 9. Diferentes recipientes plásticos y de cemento para el almacenamiento del agua lluvia.

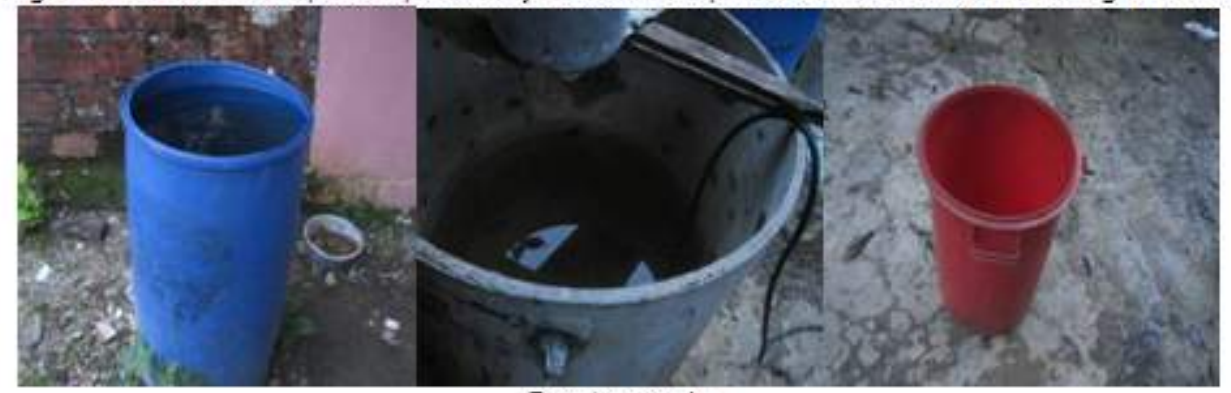

Fuente: propia.

La Figura 9 presenta envases plásticos y de cemento utilizados para almacenar agua en las diferentes viviendas del Consejo Comunitario. 
Figura 10. Pila y recipiente metálico para el almacenamiento del agua lluvia.

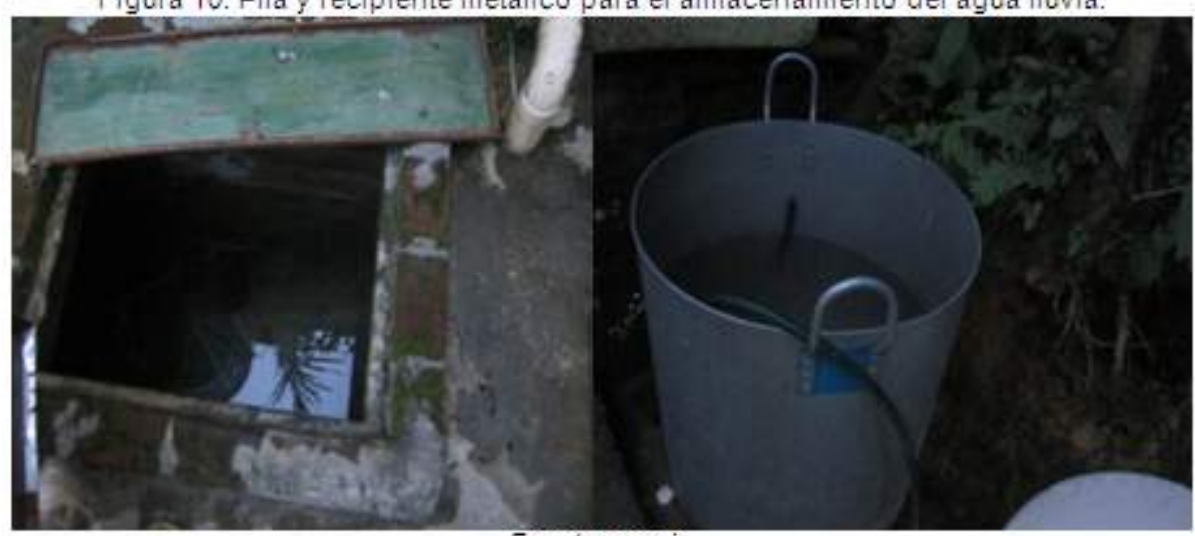

Fuente: propia

La figura 10 presenta una pila y un envase metálico para el almacenamiento del agua Iluvia, los cuales son utilizados en las diferentes viviendas del Consejo Comunitario.

A pesar de que en la comunidad se cuenta con diferentes recipientes para el almacenamiento del agua lluvia, estos no son suficientes para tener agua en las viviendas por mucho tiempo, de acuerdo como se expresa en la siguiente tabla:

Tabla 5. Capacidad de almacenamiento de agua en las viviendas

\begin{tabular}{|c|c|c|}
\hline $\begin{array}{c}\text { Tiempo de almacenamiento } \\
\text { de agua en las viviendas }\end{array}$ & $\begin{array}{c}\text { Valor en } \\
\text { porcentajes }\end{array}$ & $\begin{array}{c}\text { Cantidad de } \\
\text { agua recolectada } \\
\text { (Its) }\end{array}$ \\
\hline Maximo 1 semana & $70 \%$ & 1000 \\
\hline Maximo 2 semanas & $10 \%$ & 2000 \\
\hline Maximo 3 semanas & $5 \%$ & 3000 \\
\hline Maximo 4 semanas & $5 \%$ & 4000 \\
\hline Mas de 4 semanas & $10 \%$ & -4000 \\
\hline TOTAL & $100 \%$ & \\
\hline \multicolumn{2}{|c|}{ Fuente: propia. }
\end{tabular}

En la tabla anterior es explicito que la mayoría de las viviendas no presentan capacidad de almacenar agua por más de una semana (70\%), y tan sólo el 10\% tienen capacidad de almacenar agua hasta dos semanas; de igual forma, hasta las semanas tres y cuatro sólo se presenta una capacidad de almacenamiento del 5\% respectivamente; finalmente sólo el $10 \%$ de las viviendas tienen capacidad de almacenar agua por más de cuatro semanas y son exactamente el porcentaje de 
viviendas que tienen pilas, dado que merced a ello aumentan significativamente la capacidad de almacenar agua lluvia.

De acuerdo con lo anterior y teniendo en cuenta el número de personas promedio que habitan en las viviendas se presenta un consumo promedio de 30 litros diarios por persona; sin embargo, en algunas ocasiones se excede este consumo por lo que el agua escasea entre los tres y cinco días.

Se destaca el hecho de que en todas las viviendas, el agua lluvia captada se utiliza para todos los usos domésticos (consumo directo, preparar alimentos, lavar ropa, asear la casa, otros usos), destacando que el 90\% de las personas beben directamente el agua lluvia sin hervirla, además sólo el 15\% aplica algún tratamiento diferente de hervir el agua lluvia, lo que deja ver que el tratamiento que se le hace es muy bajo.

La bebida directa y sin ningún tratamiento del agua lluvia ha ocasionado problemas de diarrea en el $40 \%$ de la población, destacando que el $95 \%$ de ellos son niños y el $5 \%$ adultos, evidenciándose la necesidad del mejorar el aprovechamiento del agua lluvia.

Finalmente, es de destacar que en el 100\% de las viviendas consideran que es necesario mejorar el sistema de captación del agua lluvia, y están de acuerdo en mejorar el sistema de captación del agua lluvia puesto que quieren evitar los riesgos asociados al consumo de agua sin ningún tratamiento y mejorar su calidad de vida.

\subsection{Calidad del agua lluvia captada}

Los resultados de la calidad del agua lluvia captada presenta los parámetros microbiológicos y fisicoquímicos, tal como se presenta a continuación:

\subsubsection{Parámetros microbiológicos}

El comportamiento de los parámetros microbiológicos, se ilustra en la siguiente tabla: 
Tabla 6. Comportamiento de parámetros microbiológicos

\begin{tabular}{|c|c|c|c|c|c|c|c|}
\hline \multirow{2}{*}{ Tipo techo } & \multirow{2}{*}{ Parámetros } & \multicolumn{2}{|c|}{ Julio } & \multicolumn{2}{|c|}{ Agosto } & \multicolumn{2}{|c|}{ septiembre } \\
\hline & & Obtenido & Cumple & Obtenido & Cumple & Obtenido & Cumple \\
\hline \multirow{2}{*}{ Zinc } & $\begin{array}{l}\text { Coliformes } \\
\text { totales }\end{array}$ & Presente & NO & Presente & NO & Presente & NO \\
\hline & Escherichia Coli & Ausente & SI & Ausente & SI & Ausente & SI \\
\hline \multirow[t]{2}{*}{ Asbesto } & $\begin{array}{l}\text { Coliformes } \\
\text { totales }\end{array}$ & Presente & NO & Presente & NO & Presente & NO \\
\hline & Escherichia Coli & Presente & NO & Presente & NO & Presente & NO \\
\hline
\end{tabular}

No cumple requerimientos exigidos

En la tabla 6 se observa que en el techo de zinc en los tres meses, no se evidencia presencia del parámetro Escheriachia Coli, los demás parámetros evaluados están presentes en todos los meses, en ambos tipos de techo. Se resalta que el techo de zinc tiene un mejor comportamiento que el techo de asbesto, en cuanto a ausencia de microorganismos evaluados. Estos resultados indican claramente que la calidad del agua lluvia captada en la zona de estudio, en cuanto a parámetros microbiológicos se refiere, se cumple solo en forma parcial por las viviendas que captan agua lluvia con techos de zinc.

La presencia de coliformes totales en el agua lluvia estudiada, indica que existe contaminación por diferentes bacterias representando ello riesgo para la salud humana. Esta contaminación es muy generalizada debido a que puede ser humana, animal, de los suelos y otras fuentes; por lo que en nuestro caso de estudio puede obedecer a fuentes de origen animal (incluyendo, ratas, cucarachas y otros insectos) y ambientales dado que en la mayoría de las viviendas no se realiza limpieza de los elementos del sistema de captación del agua lluvia adecuado; además, los recipientes donde se almacena esta no tiene una adecuada protección con el medio exterior.

Por último, la presencia de Escheriachia Coli está relacionada directamente por la contaminación fecal de origen animal o humano, indicando ello que los techos de asbesto son más susceptible a este tipo de contaminación (reportó presencia) que los techos de zinc (no reportó presencia).

Respecto a los parámetros fisicoquímicos evaluados para el periodo de estudio, a continuación se presentan los resultados obtenidos. 


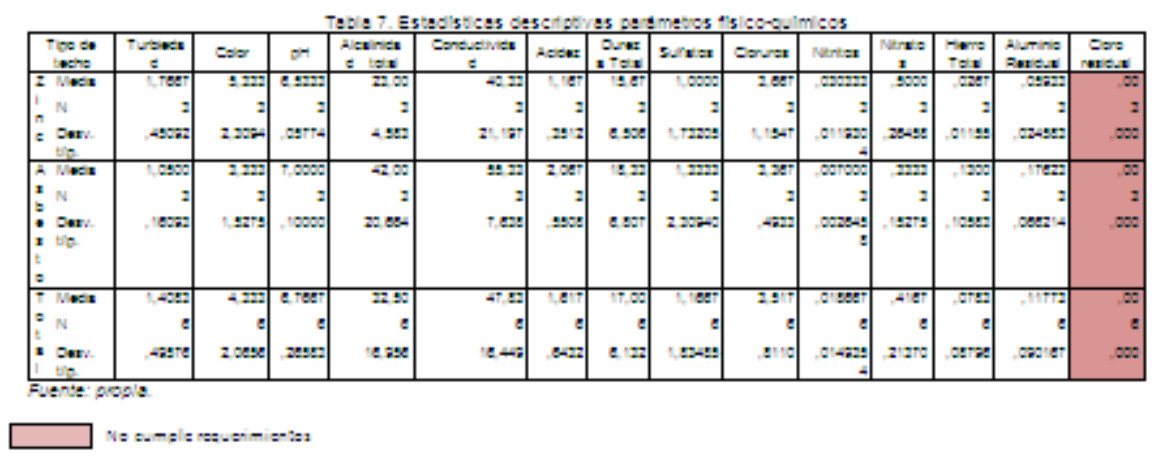

Haga clic sobre la imagen para ampliarla

De acuerdo con la estadística descriptiva, los promedios obtenidos establecen que en los dos tipos de techos (zinc y asbesto) se cumple con los requerimientos exigidos por el decreto 1575 de 2007 y la resolución 2115 de 2007, a excepción de cloro residual.

Por su parte, de acuerdo al análisis de varianza (tabla 8a, b, c y d), se concluye que a un nivel de significancia del $5 \%$, no hay diferencias significativas entre los parámetros físico-químicos evaluados en los dos tipos de techo: zinc y asbesto. Solamente hay diferencias significativas en los parámetros: $\mathrm{pH}$, Nitritos y aluminio residual; de estos, el pH y Nitritos es más favorable en techos de asbesto, y el aluminio residual es más ventajoso en techo de zinc. 
Tabla 8a. Análisis de varianza (Anova) para probar la hipótesis de que no hay Diferencias significativas entre los tipos de techo para cada uno de los Factores físico-químicos evaluados en el agua lluvia captada.

\begin{tabular}{|c|c|c|c|}
\hline Parámetro & $\begin{array}{c}\text { Fuente de } \\
\text { variación }\end{array}$ & $\begin{array}{c}\text { Suma de } \\
\text { cuadrados }\end{array}$ & Sig. \\
\hline \multirow{3}{*}{ Turbiedad } & Inter-grupos &, 770 &, 061 \\
& Intra-grupos &, 458 & \\
& Total & 1,229 & \\
\hline \multirow{3}{*}{ Color } & Inter-grupos & 6,000 &, 279 \\
& Intra-grupos & 15,333 & \\
\hline \multirow{3}{*}{ pH } & Total & 21,333 & \\
& Inter-grupos & 3,227 &, 000 \\
& Intra-grupos &, 053 & \\
& Total & 3,280 & \\
\hline
\end{tabular}

Fuente propia.

Continuación tabla 8b. Análisis de varianza factores físico-químicos

\begin{tabular}{|c|c|c|c|}
\hline Parametro & $\begin{array}{l}\text { Fuente de } \\
\text { variación }\end{array}$ & $\begin{array}{l}\text { Suma de } \\
\text { cuadrados }\end{array}$ & Sig. \\
\hline \multirow{3}{*}{$\begin{array}{l}\text { Alcalinidad } \\
\text { total }\end{array}$} & Inter-grupos & 541,500 & \multirow[t]{3}{*}{195} \\
\hline & Intra-grupos & 896,000 & \\
\hline & Total & 1437,500 & \\
\hline \multirow{3}{*}{ Conductividad } & Inter-grupos & 337,500 & \multirow[t]{3}{*}{, 313} \\
\hline & Intra-grupos & 1015,333 & \\
\hline & Total & 1352,833 & \\
\hline \multirow[b]{2}{*}{ Acidez } & Inter-grupos & 1,215 & \multirow[t]{2}{*}{, 075} \\
\hline & Intra-grupos & $\begin{array}{c}853 \\
2068\end{array}$ & \\
\hline \multirow{3}{*}{ Dureza Total } & Inter-grupos & $\begin{array}{l}2,000 \\
10,667\end{array}$ & \multirow{3}{*}{.649} \\
\hline & Intra-grupos & 177,333 & \\
\hline & Total & 188,000 & \\
\hline
\end{tabular}

Fuente propia. 
Continuación tabla 8c. Análisis de varianza factores físico-químicos

\begin{tabular}{|c|c|c|c|}
\hline Parámetro & $\begin{array}{c}\text { Fuente de } \\
\text { variación }\end{array}$ & $\begin{array}{c}\text { Suma de } \\
\text { cuadrados }\end{array}$ & Sig. \\
\hline \multirow{3}{*}{ Sulfatos } & Inter-grupos &, 167 &, 851 \\
& Intra-grupos & 16,667 & \\
& Total & 16,833 & \\
\hline \multirow{2}{*}{ Cloruros } & Inter-grupos &, 135 &, 700 \\
& Intra-grupos & 3,153 & \\
& Total & 3,288 & \\
\hline \multirow{2}{*}{ Nitritos } & Inter-grupos &, 001 &, 030 \\
& Intra-grupos &, 000 & \\
& Total &, 001 & \\
\hline \multirow{3}{*}{ Nitratos } & Inter-grupos &, 042 &, 398 \\
& Intra-grupos &, 187 & \\
& Total &, 228 & \\
& \multicolumn{2}{|c}{ Ful }
\end{tabular}

Fuente propia.

Continuación tabla 8d. Análisis de varianza factores físico-químicos

\begin{tabular}{|c|c|c|c|}
\hline Parámetro & $\begin{array}{c}\text { Fuente de } \\
\text { variación }\end{array}$ & $\begin{array}{c}\text { Suma de } \\
\text { cuadrados }\end{array}$ & Sig. \\
\hline \multirow{3}{*}{ Hierro Total } & Inter-grupos &, 016 &, 168 \\
& Intra-grupos &, 023 & \\
& Total &, 039 & \\
\hline \multirow{2}{*}{ Aluminio } & Inter-grupos &, 031 &, 025 \\
Residual & Intra-grupos &, 010 & \\
& Total &, 041 & \\
\hline \multirow{2}{*}{ Cloro } & Inter-grupos &, 000 & \\
residual & Intra-grupos &, 000 & \\
& Total &, 000 & \\
\hline
\end{tabular}

Fuente: propia.

Es importante notar que de acuerdo al estadístico de Levene, el supuesto de homogeneidad de varianzas en los dos tipos de techo, para todos los parámetros físico-químicos se cumplen, la excepción son los parámetros: Nitrito y hierro total (ver tabla 9). 


\begin{tabular}{|c|c|c|c|c|}
\hline \multicolumn{5}{|c|}{ Tabla 9. Prueba de homogeneidad de varianzas } \\
\hline Parámetro & $\begin{array}{c}\text { Estadístico } \\
\text { de Levene }\end{array}$ & gl1 & gl2 & Sig. \\
\hline Turbiedad & 1,755 & 1 & 4 &, 256 \\
\hline Color & 1,241 & 1 & 4 &, 328 \\
\hline $\mathrm{pH}$ & 2,571 & 1 & 4 &, 184 \\
\hline $\begin{array}{c}\text { Alcalinidad } \\
\text { total }\end{array}$ & 3,546 & 1 & 4 &, 133 \\
\hline Conductividad & 2,305 & 1 & 4 &, 204 \\
\hline Acidez & 1,362 & 1 & 4 &, 308 \\
\hline Dureza Total &, 067 & 1 & 4 &, 808 \\
\hline Sulfatos &, 640 & 1 & 4 &, 469 \\
\hline Cloruros & 4,418 & 1 & 4 &, 103 \\
\hline Nitritos & 8,063 & 1 & 4 &, 047 \\
\hline Nitratos & 1,600 & 1 & 4 &, 275 \\
\hline Hierro total & 9,394 & 1 & 4 &, 037 \\
\hline $\begin{array}{c}\text { Aluminio } \\
\text { Residual }\end{array}$ & 4,551 & 1 & 4 &, 100 \\
\hline Cloro residual &. & 1 & $\cdot$ &. \\
\hline
\end{tabular}

Fuente: propia.

Los anteriores resultados indican que la calidad del agua lluvia captada por las viviendas de la comunidad los lagos, no cumple con los requerimientos mínimos de ausencia de microorganismos biológicos, pero si cumple la normatividad para todos los parámetros físico-químicos evaluados, sin importar el tipo de techo, su comportamiento es muy similar (no hay diferencias significativas). De esta forma, el agua captada en las viviendas con techo de zinc, tiende a presentar una mejor calidad dado que cumple con uno de los dos parámetros microbiológicos evaluados, es decir presenta ausencia de la bacteria Escherichia Coli.

\section{CONCLUSIONES Y RECOMENDACIONES}

Se recomienda que en las viviendas del Consejo Comunitario de la Comunidad Negra de Los Lagos estén dotadas de techos zinc dado que mantienen mucho más limpios que los techos de asbesto y la calidad del agua que se capta es mejor en los primeros.

El sistema de recolección de las aguas lluvias es deficiente dado que en la mayoría de las viviendas no contempla la aplicación que algún tratamiento para eliminar la contaminación por coliformes totales y fecales; por lo que se requiere identificar 
alternativas tecnológicas individuales que permitan darle tratamiento al agua captada.

Es importantes que se adopten políticas públicas conducentes a mejorar el aprovechamiento de agua lluvia en las comunidades rurales del pacífico colombiano dado que estás comunidades en su mayoría no tienen acceso al servicio público de acueducto y requieren una fuente segura para satisfacer sus necesidades relacionadas con el recurso hídrico.

Teniendo en cuenta que en las áreas rurales de la región pacífica la mayoría de las comunidades tienen el agua lluvia como su fuente principal de abastecimiento, es importante continuar investigando las mejores formas de aprovechar este recurso natural en óptimas condiciones de cumplimiento de la legislación nacional y minimización de riesgos para la salud.

\section{BIBLIOGRAFÍA}

- CENTRO internacional de DEMOSTRACIÓN Y CAPACITACIÓN EN APROVECHAMIENTO DEL AGUA DE LLUVIA. (2007). Manual sobre Sistemas de Captación y Aprovechamiento del Agua de Lluvia para Uso Doméstico y Consumo Humano. Colegio de Postgraduados de INSTITUTO DE ENSEÑANZA E INVESTIGACIÓN EN CIENCIAS AGRICOLAS. Ciudad de México, México. 151p.

- dePARTAMENTO NACIONAL DE ESTADISTICA (2013). Encuesta nacional de calidad de vida. Bogotá D.C. Recuperado de https://www.dane.gov.co/files/investigaciones/condiciones_vida/calidad_vida/cp_ECV_2012.pdf Consultado 08 de octubre de 2014.

- departamento nacional de Planeación. Consejo Nacional de Política Económica y Social (CONPES). Documento CONPES 3491. Política de Estado para el Pacífico Colombiano, Bogotá, 2007.

- Duran, Diana (2011). Material de clases del módulo los problemas ambientales y sus perspectivas de solución. Curso I Planificación ambiental. Master en proyectos ambientales Instituto Latinoamericano de Ciencias. Huancayo, Perú. 54p. 
- IDEAM. (2014). Valores totales diarios de precipitación años 2009 - 2013. Estación 53115010 APTO Buenaventura. Sistema de información nacional ambiental. Bogotá D.C.

- Guhl, E. (2007). La problemática del agua en Colombia: para que queremos el agua los colombianos. Ponencia. En Cátedra ambiental pensamiento y política ambiental. Instituto de Investigaciones Ambientales. Universidad Tecnológica de Pereira. Pereira, Colombia.

- Hurtado, Jacqueline. (2000). Metodología de la investigación holística. Edición 3ra. Servicios y proyecciones para América Latina, Instituto Universitario de Tecnología Caripito. Caracas, Venezuela. 628p.

- Hurtado, Jacqueline. (2002). El proyecto de investigación holística. Servicios y proyecciones para América Latina - Editorial Magisterio. Caracas, Venezuela. $52 \mathrm{p}$.

- MiNISTERIO dE LA PROTECCIÓN SOCIAL; MINISTERIO DE AMBIENTE, VIVIENDA Y DESARROLLO TERRITORIAL. Resolución número 2115 de 2007. Por medio de la cual se señalan características, instrumentos básicos y frecuencias del sistema de control y vigilancia para la calidad del agua para consumo humano. Bogotá D.C. 23p.

- MiNISTERIO DE LA PROTECCIÓN SOCIAL. Decreto número 1575 de 2007. Por el cual se establece el Sistema para la Protección y Control de la Calidad del Agua para Consumo Humano. Bogotá D.C. 14p.

- ORGANIZACIÓN MUNDIAL DE LA SALUD. (2006). Guías para la calidad del agua potable: incluye el primer apéndice. Vol. 1: Recomendaciones. Tercera edición. Recurso electrónico: Link http://www.who.int/water_sanitation_health/dwq/gdwq3_es_fulll_lowsres.pdf

- OGANIZACIÓN PANAMERICANA DE LA SALUD. (2010). Tecnologías apropiadas para el suministro de agua en situaciones de emergencia. Colombia. 68p. Recurso electrónico: Link: file:///D:/Downloads/tecnologiasAGUA.pdf

- Severiche S, Carlos A; Castillo B, Marlon E; Acevedo B, Leonor. (2013). Manual de métodos analíticos para la determinación de parámetros fisicoquímicos básicos en 
aguas. Fundación Universitaria Andaluza Inca Garcilaso. Cartagena de Indias, Colombia. 101p.

\section{ANEXOS}

\section{Anexo 1. Encuesta caracterización viviendas, diagnóstico situacional} captación y usos del agua Iluvia

1. Es propietario de la vivienda: sí $\mathrm{NO}$

2. Cuántas personas viven en la casa:
A) Entre 1 y 3
B) Entre 4 y 6
C) Entre 7 y 9
D) Mayor o igual a 10

3. Tiene sistema de acueducto: sí $\mathrm{NO}$

4. Cuál es la fuente más importante con que usted cuenta para abastecerse de agua:
A) Agua Iluvia
B) Río o quebrada
C) Agua subterránea

5. Realiza captación de aguas Iluvias: sí $\mathrm{NO}$

6. Los recipientes que tiene para cuanto tiempo de abastecimiento alcanzan:

A) 1 semana

B) 2 semanas

C) 3 semanas

D) 4 semanas E) Más de 4 semanas 
7. Entre los recipientes anteriores tiene piscina: $S$

NO

8. Qué tipo de techo tiene su vivienda:

A) Zinc

B) Asbesto

C) Рaja

D) Cemento

9. En que usos domésticos utiliza el agua Iluvia:

A) Beber y/o preparar alimentos

B) Lavar ropa

C) Asear la casa

D)

Otros usos

E) Todas las anteriores

10. En caso de beber el agua lluvia, previamente la hierve: sí

NO

11. En caso de beber el agua lluvia, realiza algún tratamiento diferente de hervirla:

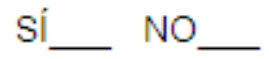

12. Se ha enfermado alguna persona por el consumo de agua lluvia:

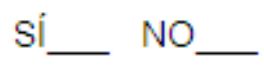

13. Qué enfermedad(es) ha ocasionado el agua lluvia: 
14. Las personas que han sufrido enfermedad por el agua lluvia son niños o adultos:

A) Niños B) Adultos

15. Cree usted que es necesario mejorar el sistema de captación de agua:

Sí $\mathrm{NO}$

16. Estaría usted de acuerdo con mejorar el sistema de aprovechamiento del agua:

Sí NO

Anexo 2. Toma de muestras de aguas lluvias por trabajadores de la empresa Hidropacífico

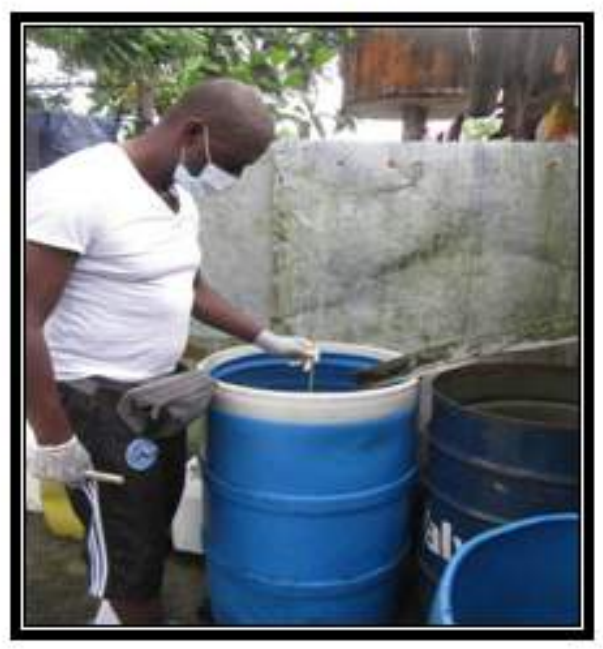

Fuente: el autor. 
Registro fotográfico 1. Medición de la temperatura del agua.

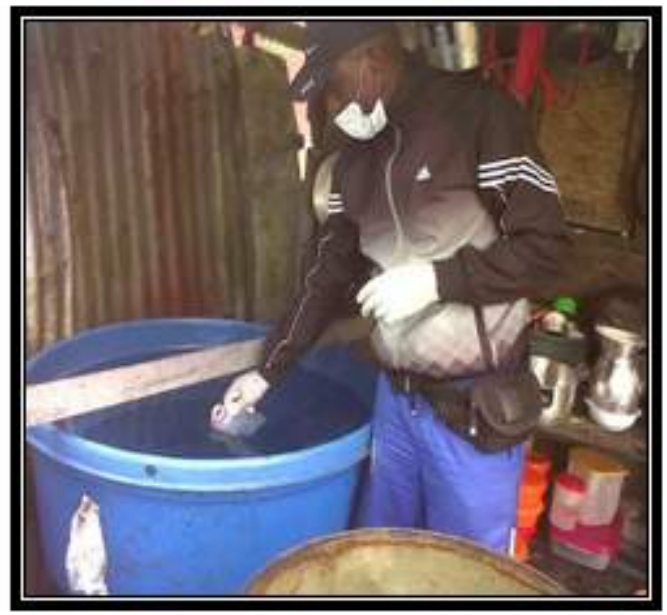

Fuente: el autor.

Registro fotográfico 2 . Toma de muestra fisicoquímica.

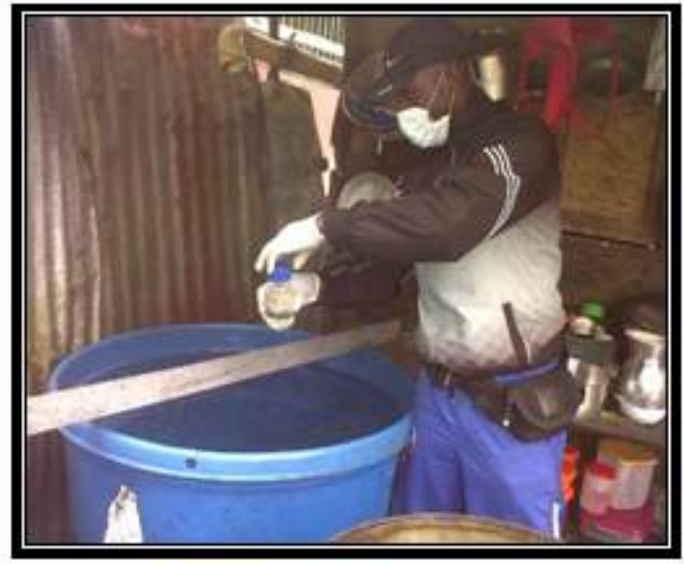

Fuente: el autor.

Registro fotográfico 3. Toma de muestra microbiológica.

'Doctorando en Ordenamiento Territorial y Desarrollo Sostenible, Magister en Desarrollo Sostenible y Medio Ambiente, Especialista en Gestión Ambiental, Administrador Ambiental (graduado como estudiante distinguido); Docente de la Universidad del Pacífico; Buenaventura, Colombia; e-mail: nixon@utp.edu.co. ORCID: 0000-0002-6769-9202 
ii Guhl (2007) destaca que la precipitación media anual del planeta es de $900 \mathrm{~mm}$, en Sur América de 1.600 mm y en Colombia es del orden de 3.000 mm, lo que hace que nuestro país tenga casi el triple de la precipitación promedio del mundo. A pesar de que Colombia es un país con un alto promedio de lluvia, en la región pacífica llueve por lo menos el doble de esa precipitación.

iii De acuerdo con la Ley 70 de 1993, actualmente el Consejo Comunitario de la Comunidad Negra Los Lagos está tramitando ante el INCODER, su título colectivo del territorio, ya que cumplen con lo establecido en el artículo $1^{\circ}$ de la ley en mención.

iv La empresa Hidropacífico E.S.P. S.A. es la empresa prestadora del servicio de acueducto en el distrito de Buenaventura y cuenta con un laboratorio certificado por el IDEAM.

${ }^{v}$ Los datos de precipitación corresponden a los resultados obtenidos entre los años 2009 y 2013 en la estación meteorológica que tiene el Instituto de Hidrología, Meteorología y Estudios Ambientales en el aeropuerto de Buenaventura, el cual está ubicado a una distancia aproximada de $1 \mathrm{Km}$. de la zona de estudio.

Para citar este artículo: Arboleda-Montaño, N. Diagnóstico del sistema de aprovechamiento del agua lluvia en el consejo comunitario de la comunidad negra de Los Lagos, Buenaventura.Revista Luna Azul, 43, 29-55. Recuperado de http://200.21.104.25/lunazul/index.php?option=com_content\&view=article\&id=192 